\title{
Gradhiva
}

\section{Carlo Severi, Le Principe de la chimère : une}

anthropologie de la mémoire

Paris, éditions Rue d'Ulm-musée du quai Branly, coll. " Aesthetica », 2007, 370 p.

\section{Stéphanie Leclerc-Caffarel}

\section{(2) OpenEdition}

Journals

Édition électronique

URL : http://journals.openedition.org/gradhiva/1777

DOI : $10.4000 /$ gradhiva. 1777

ISSN : 1760-849X

Éditeur

Musée du quai Branly Jacques Chirac

Édition imprimée

Date de publication : 19 mai 2010

Pagination : 230-232

ISBN : 978-2-35744-025-8

ISSN : 0764-8928

Référence électronique

Stéphanie Leclerc-Caffarel, "Carlo Severi, Le Principe de la chimère : une anthropologie de la mémoire », Gradhiva [En ligne], 11 | 2010, mis en ligne le 09 mai 2010, consulté le 21 septembre 2020. URL : http:// journals.openedition.org/gradhiva/1777 ; DOI : https://doi.org/10.4000/gradhiva.1777

Ce document a été généré automatiquement le 21 septembre 2020.

(c) musée du quai Branly 


\section{Carlo Severi, Le Principe de la} chimère : une anthropologie de la

\section{mémoire}

Paris, éditions Rue d'Ulm-musée du quai Branly, coll. " Aesthetica », 2007, 370 p.

\section{Stéphanie Leclerc-Caffarel}

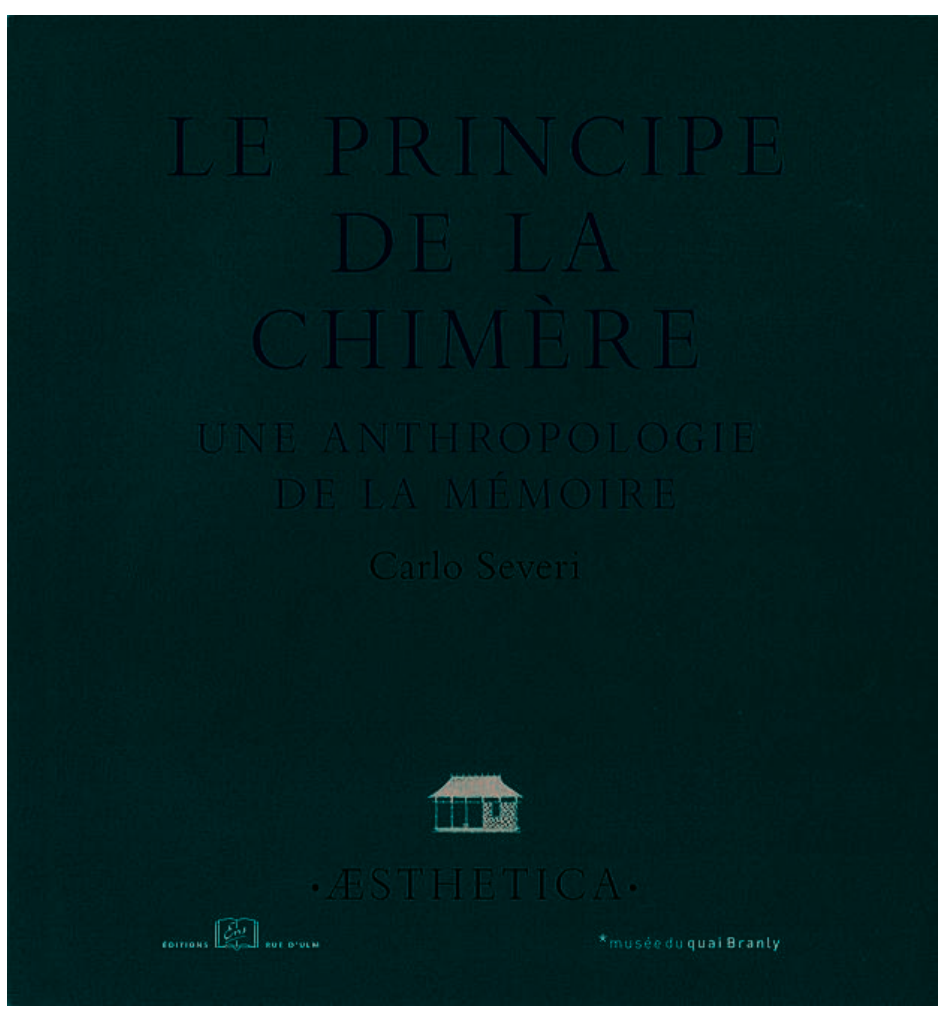

1 Le Principe de la chimère est le fruit d'années de recherches et de réflexions. Cas ethnographiques à l'appui, l'anthropologue Carlo Severi s'interroge et donne matière à réfléchir sur une possible définition d'un «art de la mémoire» dans les sociétés dites 
"sans écriture ». Se retrouvent dans cet ouvrage les thèmes de prédilection de l'auteur: le pouvoir des images, la mémoire, les spécificités du contexte rituel, les fonctions cognitives, la discipline anthropologique, etc. De même, le dialogue avec ses maîtres, Claude Lévi-Strauss et Aby Warburg notamment, se poursuit. Sont également présents ses travaux précédents, études de cas océaniens et, surtout, américains. Ce qui change : l'objectif et la méthode. Cette fois, Carlo Severi utilise ces matériaux dans une perspective synthétique et comparative de généralisation.

2 Au départ de ce projet intellectuel est le constat d'un préjugé récurrent : l'écrit serait le seul biais du maintien et de la transmission de connaissances cohérentes et partagées. Dans cette perspective, "toute mémoire orale est, inévitablement, mémoire de quelqu'un ", individuelle et arbitraire, vouée à l'altération. Comment, dès lors, pourrait se constituer une mémoire sociale, un socle commun de références dans les sociétés de " tradition orale »? Il y a dans cette interrogation une double faille que souligne Carlo Severi. D'abord, l'adjectif «orale » appliqué au terme de «tradition» est trop vague pour être pertinent. Il omet notamment de distinguer les deux formes principales de l'oralité : la narration et la récitation rituelle. Ensuite, la désignation «tradition orale » ne rend compte de la réalité que partiellement. De fait, souvent, de telles traditions sont aussi « iconographiques ».

3 Ce vocabulaire qui peine à définir avec pertinence les différences culturelles est un problème récurrent de l'anthropologie. Carlo Severi s'y arrête un moment, rappelant que la posture ethnocentrique conduit à penser le monde selon une logique binaire, en termes de différences entre "Eux et Nous ", à catégoriser "l'Autre » seulement par rapport à ce qu'il n'est pas. Ce procédé de définition négative est particulièrement évident en ce qui concerne les sociétés dites "sans écriture », " orales ». L'auteur trouve une alternative à cet ethnocentrisme dans la multiplication des systèmes référents et dans une analyse comparative procédant de proche en proche. L'enjeu du Principe de la chimère : montrer comment l'articulation de l'image et de la parole peut devenir, en contexte rituel, support et outil de mémoire sociale.

Dans un premier chapitre que l'on pourrait concevoir en deux parties, dense mais bien mené, Carlo Severi donne les clés de compréhension de son " principe de la chimère ». À la recherche de précédents, de pistes de réflexion et d'outils méthodologiques, il explore l'histoire du questionnement de la relation image-parole-mémoire. Cela, d'abord, lui permet de souligner un des nœuds du problème. Fort longtemps, on a considéré les développements iconographiques des sociétés «orales» comme de simples ornements ou comme des tentatives manquées d'écriture. Cette stricte opposition écriture-non écriture relève d'une "symétrie fallacieuse ». Elle est inapte à décrire la pluralité des situations intermédiaires dans lesquelles la relation imageparole-mémoire est véhicule de sens et garante d'un savoir commun. Ce sont ces fonctionnements mnémoniques, ces dispositifs cohérents et efficaces, totalement différents de la codification phonétique du langage, qu'il s'agit de mettre en lumière ici.

Dans l'œuvre «à la fois fertile et inaccomplie » de Warburg, Carlo Severi perçoit un préambule à sa réflexion. Dans l'entourage intellectuel de l'historien de l'art allemand, il trouve des pistes méthodologiques. Le concept de «biologie des images », élaboré par le général anglais Pitt-Rivers et développé notamment par le Suédois Hjalmar Stolpe dans la seconde moitié du XIXe siècle, lui semble fécond. Complétée par l'approche morphologique d'origine allemande (goethéenne), cette « biologie » permet d'envisager 
une anthropologie comparative des formes iconographiques supports de mémoire, réparties, à chaque étape, selon une échelle de complexité croissante.

Dans cette perspective, ce sont les formes élémentaires de l'articulation image-parolemémoire qui priment. Elles inaugurent la seconde partie du chapitre. Dans la région du fleuve Sepik, en Nouvelle-Guinée, des supports visuels codifiés (corde à nœuds, crochets) permettent de mémoriser des listes de noms propres. Ces exemples, et celui de l'oiseau-serpent hopi extrait de «l'ethnographie » warburgienne, permettent à Carlo Severi de formuler son "principe de la chimère ». Deux critères rendent une image mémorable: l'ordre et la saillance. Le premier principe est tel que les références visuelles qui composent une représentation graphique doivent être organisées en « images-séquences » signifiantes. Cet ordonnancement des indices visuels permet le déchiffrement et l'interprétation de l'image, l'opération mentale qui fait lien avec la mémoire. Le second principe engendre celui « d'objet-chimère ». Une image ne peut être mémorable que si elle se différencie de la perception quotidienne. Pour ce faire, elle incorpore des traits hétérogènes. Dans leur contexte rituel d'apparition, ces figures, qui pourraient paraître vides de sens ailleurs, deviennent des complexes d'indices visuels qui doivent être élaborés mentalement.

7 Cette élaboration mentale est l'objet du deuxième chapitre. L'auteur y définit ce que sont les pictographies amérindiennes et les utilise pour démontrer l'efficacité d'un dispositif mnémonique : le " parallélisme ». Le lecteur entame alors un voyage à travers les traditions religieuses américaines qui se poursuit jusqu'à la fin de l'ouvrage, de l'Amazonie au Grand Nord et depuis la préhistoire jusqu'aux époques contemporaines. Dans ce chapitre, Carlo Severi démontre en quoi les pictographies amérindiennes constituent non pas un ornement, non pas une proto-écriture, mais à proprement parler «un art de la mémoire ». Les systèmes ici comparés révèlent une grande cohérence interne, une étonnante aptitude à persister dans le temps et une large diffusion géographique, au-delà des variations stylistiques. La mnémotechnique paralléliste repose sur une construction parallèle - mais non symétrique - des textes et des images, sur la combinaison des traces mnésiques sonores et visuelles en une sorte de mosaïque mentale. Des formules répétées, constantes, relèvent de la seule énonciation, tandis que les variations qui s'ajoutent sur ce canevas redondant sont, elles, figurées par des pictographies, ordonnées et saillantes.

8 Les deux derniers chapitres se penchent sur une autre fonction de l'articulation mentale image-parole-mémoire : l'évocation. L'évocation est ce qui lie l'énonciateur et le destinataire du rituel et, par là même, induit la croyance. L'image n'est plus seulement support mnémonique, elle devient projection. Un symbolisme partagé permet à la parole énoncée de faire sens dans l'esprit du destinataire. Jouant sur cette orientation de l'imagination, l'énonciateur rituel se fait lui-même chimère. Le chamane kuna, par exemple, pratique un parallélisme réflexif pour accumuler, par métamorphoses successives, des identités hétérogènes, souvent contradictoires. L'identité symbolique, temporaire et précaire du chamane pendant le rituel crée une tension avec la perception quotidienne. Celle-ci laisse place au doute inhérent à la croyance que Carlo Severi conçoit comme une impossibilité à ne pas croire. L'évocation est donc la clé de l'efficacité de certains rituels et le moyen d'une communication « infraverbale ». Elle permet de rendre présents l'indicible, le surnaturel, mais aussi la douleur, la peur, l'inconnu, l'hostile, etc. 
9 C'est l'interrogation de cette assimilation qui clôt la réflexion ici amorcée. Dans le contexte spécifique $d u$ conflit culturel, dénominations et images reflètent la confrontation de deux cultures, ennemies mais mutuellement "contagieuses». La relation image-parole-mémoire se fait ici politique. De nouvelles « chimères » naissent de ces tensions. Elles témoignent de l'évolution historique de la mémoire sociale, de son intégration du conflit. Elles permettent aussi à Carlo Severi de rappeler une chose essentielle : l'oubli est constitutif de toute construction mnémonique.

Très académique, Carlo Severi utilise avec pertinence des exemples ethnographiques classiques mais passionnants. L'ouvrage est clair et pédagogique. Les références sont abondantes et le propos bien documenté. Globalement, la démonstration fonctionne. Elle nous mène même bien plus loin que le titre de l'ouvrage ou la quatrième de couverture ne pouvaient le laisser supposer. Si certains passages sont un peu laborieux à la lecture, d'autres en revanche sont lumineux. Le procédé d'analyse, répété à chaque chapitre, permet d'enrichir progressivement la compréhension de la relation imageparole-mémoire. Une approche méthodologique qui se limite, toutefois, au contexte rituel. La restriction est cependant volontaire, elle assure l'acuité de la démonstration. Issu d'un travail de recherche en cours, l'ouvrage n'a pas vocation d'exhaustivité. On peut néanmoins espérer que la réflexion se poursuive. La distinction narrationrécitation rituelle, présupposé de la démarche, n'est par exemple que peu explicitée. De même, si Carlo Severi use avec habileté d'autres champs du savoir, tels que la représentation théâtrale et la psychanalyse, en matière d'anthropologie de l'art, en revanche les travaux d'Alfred Gell, bien que controversés, auraient sans doute pu trouver ici une place.

11 Finalement, les questionnements sont nombreux et invitent à poursuivre la réflexion, notamment à confronter l'analyse à d'autres exemples d'art non occidental. Il y a indéniablement dans Le Principe de la chimère matière à penser.

\section{AUTEUR}

\section{STÉPHANIE LECLERC-CAFFAREL}

S.Leclerc-Caffarel@uea.ac.uk 